\title{
Zur Arbeit mit Video
}

\author{
Von Anton Täubl
}

Video macht den Einsatz anderer audiovisueller Medien (AVM), etwa den Gebrauch von Lichtbildern oder von 16-mm-Filmen, nicht überflüssig! Abgestimmt auf vorhandene Arbeitsmöglichkeiten, orientiert an den Bedürfnissen der jeweiligen Adressaten und mit Rücksicht auf Zielsetzungen der sozialen, pädagogischen oder didaktischen Bemühungen kann Video jedoch als mediales Kommunikations- und Darstellungsmittel im schulischen und außerschulischen Freizeit- und Bildungsbereich effektiv und sinnvoll verwendet werden.

\section{Chancen und Grenzen von Video}

Eine Auflistung der Qualitäten beziehungsweise Mängel von Video getrennt nach technischen, ökonomisch-organisatorischen, pädagogisch-didaktischen und sozialkommunikativen Aspekten vorzunehmen, fällt schwer, weil sich sowohl Vorzüge als auch Schwächen des Videosystems als grenzüberschreitend erweisen und sich gegenseitig bedingen.

\subsection{Möglichkeiten von Video}

Video erschließt als audiovisuelles Medium neue kommunikative, pädagogische und didaktische Möglichkeiten und bietet in den verschiedenen Erziehungs- Bildungs- und Freizeitbereichen folgende Vorteile:

a. Der Einsatz von Video schafft keine außergewöhnliche Situation. Bild- und Tonwiedergabe entsprechen vielmehr der Alltagserfahrung vieler Betrachter von Fernsehprogrammen. Sogar die Wohnzimmeratmosphäre bleibt weithin erhalten und erfordert von den Teilnehmern kaum eine größere Umstellung (was von vielen Erziehern und Bildungsmultiplikatoren allerdings nicht nur als Vorteil angesehen wird).

b. Video ist ein Tageslichtmedium: In nichtverdunkelten Räumen sind während der Vorführung Aktivitäten möglich, wie zum Beispiel das Beobachten von Teilnehmern oder Anfertigen schriftlicher Notizen.

c. Video bietet bei der Arbeit in kleineren Gruppen mit maximal bis zu 15 Teilnehmern, für die die Qualität und Bildschirmgröße des Fernsehmonitors ausreichen, gute Präsentationsmöglichkeiten. Videoverwendung in größeren Gruppen ist von der Empfangssituation her sowohl unter wahrnehmungs- und lernpsychologischen, als auch unter filmästhetischen Gesichtspunkten äußerst problematisch und dürfte erst sinnvoll werden, wenn preisgünstige Bildschirme in Höhe und Breite von Filmleinwänden und ohne Bildverzerrung auf den Markt kommen.

Anton Täubl ist Produktionsreferent am Institut für Film und Bild in Wissenschaft und Unterricht (FWU), Grünwald/München 
d. Video ist technisch und organisatorisch leicht handhabbar, wenn die Anlage einmal steht und funktioniert. Zumindest beim Abspielen von Kassetten sind die Geräte einfach zu bedienen. Hinzu kommt die Mobilität der Anlage. Der Recorder allein ist nicht schwer und läßt sich gut tragen. Sind Fernsehgerät und Videorecorder auf einem Fahrgestell montiert und miteinander verbunden, so ist der Transport auf gleicher Ebene auch nicht umständlicher als die Arbeit mit dem 16-mm-Filmprojektor. Schwierig bleibt der Standortwechsel mit zwei Geräten von einer Etage zur anderen, wenn kein Aufzug benutzt werden kann.

e. Weitere Vorteile, die sich didaktisch und pädagogisch nutzen lassen:

- Kurze Zugriffszeiten durch schnelles Ein- und Ausschalten: Videokassetten können an jeder beliebigen Stelle rasch ausgewechselt werden; wird die Vorführung unterbrochen, ist kein zeitraubendes Rückspulen erforderlich wie beim Film.

- Der Suchlauf mit Bild ermöglicht ein rasches Auffinden gewünschter Szenen. Schnellerer Vor- und Rücklauf als bei der Filmprojektion erleichtern Wiederholungen, wodurch schwer verständliche Teile gründlicher erschlossen werden können. Damit wird nicht nur der Weg zu einer intensiveren stofforientierten und an individuelle Seh- und Lernbedürfnisse angepaßte Auseinandersetzung mit den Bildungsinhalten eröffnet, sondern auch die gemeinsame Analyse filmischer Formen ermöglicht: So können lange Videobänder wie etwa „Septemberweizen“ von Peter Krieg in Langzeitprojekte eingebunden und auf mehrere Bildungsabschnitte oder Unterrichtsstunden verteilt eingesetzt werden.

- Individuell steuerbarer Bildverlauf, zum Beispiel durch Standbildverlängerung, ermöglicht die Arbeit mit dem Einzelbild nach beliebiger Dauer. Zeitlupen- und Zeitraffer-Vorführungen begünstigen ein genaueres Betrachten: dieser „Lupeneffekt“ gestattet es den Teilnehmern, schwer erkennbare Vorgänge, wie etwa rasch ablaufende Bewegungen im Sportunterricht, durch Verlangsamen beziehungsweise Einfrieren des Bildlaufs zu verdeutlichen. Entwicklungen, die sich über einen längeren Zeitraum hinziehen, etwa Wetterbeobachtungen, können dagegen durch beschleunigtes Vorführen in konzentrierter Form übersichtlich dargeboten werden.

f. Im Unterschied zur Projektion von 16-mm-Filmen kommt es beim Video-Einsatz zu keinen störenden Nebengeräuschen. Der technisch ausgereiftere Magnetton des Videosystems erleichtert das Hörverstehen im Vergleich zum Lichtton beim Film, was bei Sprach- und Musiksendungen oder auch bei Unterrichtsdokumenten von besonderer Bedeutung ist. Das Videoband bietet darüber hinaus auch noch eine zweite Tonspur, die pädagogisch-didaktisch genutzt werden kann, etwa für einen deutschen Kommentar neben der Originalsprache bei Videoproduktionen ausländischer Herkunft oder für filmkritische Bemerkungen zu einem Spielfilm.

g. Das Videosystem schafft auch genügend Anreize zu eigener Kreativität. Interessante Fernsehsendungen können zur persönlichen Verwendung jederzeit bequem aufgezeichnet, beliebig lange gespeichert und nach Bedarf (im privaten Bereich) erneut abgespielt werden. Ist ein zweiter Recorder vorhanden, so können bereits aufgezeichnete Videoprogramme ohne allzu große Verluste in Bild- und Tonqualität leicht und billig auf ein anderes Videoband überspielt werden. Da Videobänder immer wieder gelöscht und neu bespielt werden können, ohne daß es zu merklichem Materialverschleiß kommt, eignen sie sich vor allem zur Aufzeichnung „aktueller“ Themen und von Eigenproduktionen, die nicht für eine Archivierung vorgesehen sind. 
Zur kostengünstigen Eigenherstellung von Videoproduktionen im Sinne einer ,aktiven"Medienarbeit eignen sich vor allem tragbare Videoanlagen. Magnetbänder und Videokameras sind ziemlich lichtempfindlich, so daß ohne aufwendige Beleuchtungstechnik meistens mit Tageslicht gearbeitet werden kann. Bild und Ton werden gleichzeitig aufgenommen, wodurch es nicht zu asynchronen Verschiebungen kommt wie manchmal bei nachlässig bearbeiteten Filmkopien. Aufgezeichnete Szenen können bereits während der Arbeit kontrolliert und bei Bedarf erneut aufgenommen werden, ohne daß kostbares Material verlorengeht. Zwischen Aufnahme und Wiedergabe gibt es praktisch keine zeitliche Verzögerung: Das Videoband ist unmittelbar nach den Aufzeichnungen abspielfertig, das heißt vorführbereit, weil es nicht wie das Filmmaterial noch entwickelt werden muß.

h. Ein nicht zu unterschätzender Vorteil ist zweifellos auch der erschwingliche Anschaffungspreis für das Videosystem, zumindest so lange es nur um die Aufzeichnung von Fernsehsendungen und um das Vorführen bereits bespielter Kassetten geht: Preise für Fernsehbildschirm und Videorecorder zusammen liegen derzeit kaum noch über den Kosten für gute 16-mm-Filmprojektoren; ist ein Fernsehgerät bereits vorhanden, genügt die Beschaffung des Recorders, was die Ausgaben erheblich senkt. Beim Erwerb von unbespielten wie von bespielten Videokassetten ist der Preisvorteil gegenüber 16-mm-Film-Kopien noch augenscheinlicher: Eine bespielte Videokassette mit bis zu 30 Minuten Laufzeit kostet derzeit beim Institut für Film und Bild (FWU) DM 96,- oder DM 105,-; ein Farbfilm gleicher Länge kostet dagegen rund DM950,-.

i. Ein letzter Gesichtspunkt: Platzsparende Lagerung, geringer Verpackungsaufwand und verminderte Portokosten beim Versand vereinfachen Archivierung, Lagerung und Verleih des Videomaterials, insbesondere auch für Ausleihstellen von audiovisuellen Medien.

\subsection{Vorbehalte gegenüber Video}

Trotz der genannten Vorteile äußern sich Erzieher, Pädagogen und Bildungsreferenten aller Art, auch Geistliche, oft skeptisch gegenüber dem Videosystem und nehmen eine teils abwartende, teils sogar ablehnende Haltung ein.

a. Häufig gibt es Vorwürfe im Zusammenhang mit der technischen Ausstattung, oft verbunden mit Reserven bezüglich der Akzeptanz des Videosystems überhaupt:

- Immer wieder wird vermerkt, daß sich die Arbeit mit Video (noch) nicht lohne, weil die gerätetechnische Ausstattung (hardware) und damit die Abspielbasis zu gering sei; auch stünden für Unterricht, Bildung und kirchliche Gemeindearbeit nicht genügend Videoprogramme (software) zur Verfügung. In der Tat, ohne technische Infrastruktur ist die Arbeit mit audiovisuellen Medien, und damit auch mit Video, natürlich nicht möglich; dieser Mangel an gerätetechnischer Ausstattung läßt sich jedoch beheben und darf meines Erachtens als vorübergehende Erscheinung angesehen werden. Eine ähnliche Schlußfolgerung gilt auch für die Bereitstellung von Videokassetten: Bereits jetzt gibt es ein erstaunlich reichhaltiges Angebot in allen größeren Videotheken und Videoshops für Unterhaltung, Hobby und Beratung, wie zum Beispiel in Gesundheits- und Lebensfragen; eine Reihe von Verlagen sowie das Katholische Film-Werk (Frankfurt), die Evangelische Matthias-Film (Stuttgart) und 
schließlich auch Einrichtungen wie das Institut für Film und Bild (FWU, Grünwald) bieten in letzter Zeit in verstärktem Umfang Videokassetten für den Erziehungs- und Bildungsbereich an.

- Der rasche Fortschritt zu immer kleineren und leichteren Systemen bei gleichbleibender oder sogar verbesserter Leistungsfähigkeit läßt kostspielige audiovisuelle Geräte schnell veralten. Mangelnde Standardisierung im Aufnahme- und Abspielbereich sowie Unsicherheit bezüglich der Frage nach einem langfristig geltenden Videosystem verhindern derzeit zweifellos Investitionen zur Verbesserung der Videoausstattung im Freizeit- und Bildungsbereich.

- Auch wenn Videogeräte immer benutzerfreundlicher konstruiert werden und die Störanfälligkeit stark zurückgegangen ist, so fehlt es doch oft an entsprechender Ausbildung für den Umgang im Gebrauch des Systems. Manche Erzieher, Lehrer, Bildungsreferenten und Pfarrer haben auch gar nicht mehr vor, sich umzustellen und sich mit der neuen Videotechnik vertraut zu machen.

- Einen weiteren Unsicherheitsfaktor für Videoarbeit in der Praxis bilden die zum Teil noch ungeklärten juristischen Fragen des Urheberrechts und Leistungsschutzes sowie des Jugendschutzes. Probleme der Aufzeichnung von Fernsehsendungen und der Überspielung von Videoprogrammen sowie ihre öffentliche gewerbliche oder nichtgewerbliche Vorführung gehören zu diesem Fragenkreis.

b. Systembedingte Grenzen des Videosystems und Finanzierungsprobleme, die Auswirkungen auf Verwendung in Unterricht und Bildung haben, sind ebenfalls Anlaß für Reserve und Kritik:

- Hauptnachteil von Video ist die Festlegung des Betrachters auf das kleine Fernsehbild, das bislang eine sinnvolle Videoarbeit nur in relativ kleinen Gruppen, bis etwa 15 Teilnehmern ermöglicht. Bei normaler Klassenstärke ist schon mehr als ein Sichtgerät erforderlich, was die Investitionskosten erhöht. Bei (Kino-) Filmen, die ihre filmästhetischen Qualitäten nur in verdunkeltem Raum und auf einer großen Leinwand entfalten können, geht ein Großteil der Wirkung auf dem kleinen Bildschirm verloren: Eindrucksvolle Totalen auf der Leinwand, etwa Massenszenen oder Landschaftsaufnahmen, verkümmern bisweilen auf dem Fernsehmonitor zu einer kläglichen, undefinierbaren Kulisse.

- In der Erziehungs- und Bildungsarbeit vor Ort weisen zahlreiche Videoproduktionen derzeit oft noch technische, filmästhetische und didaktische Mängel auf. Tonund Bildqualität (Farbe, Kontraste, Schärfe, Stabilität des Bildes und so weiter) sind manchmal unzureichend, vor allem bei nachlässig durchgeführten Mitschnitten von Fernsehsendungen oder beim Überspielen von Videoprogrammen. Viele der bespielten Videokassetten im Angebot haben zu lange Laufzeiten für die Arbeit in Klassen oder Gruppen, sofern im Anschluß an die Vorführung noch ein Gespräch geplant ist. Ein großer Teil des Videoangebots, vor allem Übernahmen von Fernsehproduktionen, besteht zudem aus „geschlossenen“ Einheiten, die didaktisch wenig ergiebig sind. Noch fehlen im Videoangebot derzeit weithin spezielle Programme für Unterricht und Bildung, die zum Weiterdenken anregen und zur Auseinandersetzung reizen.

- Für viele Schulen, Bildungshäuser und Gemeindezentren ist in der augenblicklichen wirtschaftlichen Lage die Anschaffung von Video-Einheiten in ausreichender Zahl nur schwer finanzierbar. Zwar wurden Videoanlagen in letzter Zeit immer 
bedienungsfreundlicher und robuster, sie bleiben aber dennoch störanfällig. Da es sich hierbei um eine komplizierte Elektronik handelt, müssen Reparaturen meistens von Fachleuten vorgenommen werden, was die Wartungskosten hochtreibt. Das gilt vor allem für tragbare Videoanlagen zur Film-Aufnahme sowie für entsprechende Bearbeitungsgeräte wie Schnittrecorder, Tonmischanlage und Tricktisch.

- Das Abstimmen des Fernsehers mit der Modulatorfrequenz des Videorecorders wird von Bildungsmultiplikatoren vielfach als problematisch erlebt, insbesondere wenn in einer Bildungsstätte mehrere Videorecorder mit unterschiedlichen Frequenzen in Gebrauch sind, etwa VHS, U-matic oder Betamax. Der Einfachheit halber wird deshalb ein Recorder meistens fest dem Fernseher zugeordnet und mit diesem passend zusammengeschaltet.

- Der Erwerb einer tragbaren Videoanlage mit dazu passenden Bearbeitungsgeräten für die Selbstherstellung von Videofilmen im Amateurbereich ist doch relativ teuer. Schnitt und Vertonung eigener Aufnahmen auf Amateur; und vor allem aber auf semiprofessioneller Basis sind zeitintensiv und erfordern präzises Arbeiten. Die Herstellung von weiteren Videokopien durch Überspielen ist dagegen einfach und preisgünstig.

c. Vorbehalte und Kritik aus erzieherischer und gesellschaftlicher Sicht gegenüber den Entwicklungen im Videobereich werden ebenfalls geäußert. Unter anderem heißt es:

- Mit Video werde die Technisierung von Erziehung und Bildung weiter vorangetrieben und eine „Entseelung“ des Lehrens und Lernens sowie der kirchlichen Verkündigungsarbeit begünstigt. Wichtiger als die Einführung neuer Techniken im Erziehungs- und Pastoralbereich bleibe die Entwicklung, Förderung und Verbesserung der personalen Kommunikation und der zwischenmenschlichen Beziehungen.

- Ferner wird gesagt, daß Video die Inflation von Ton und Bild verstärke und dadurch insbesondere bei Kindern und Jugendlichen zu einer Informationsüberflutung führe, die nicht mehr orientiere, sondern desorientiere und verunsichere. Reizfülle begünstige die Verführung zu oberflächlicher Betrachtungsweise und verstärke das passive Konsumverhalten; kreative Kräfte des Individuums verkümmerten, soziale Kontakte würden eingeschränkt, die Vereinsamung nehme zu.

- Videovorführungen in Unterricht, Bildung und kirchlicher Gemeindearbeit können als Fortsetzung des häuslichen Fernsehkonsums empfunden werden. Bei den Betroffenen werde tendenziell eine passiv-unkritische Verbraucherhaltung gefördert. Die "Wohnzimmeratmosphäre" des familiären Gewohnheitsfernsehens wirke oft eher störend als lern- und kommunikationsfördernd.

Der Abnutzungseffekt von Video mit negativen Auswirkungen auf das Lehren und Lernen dürfe jedenfalls nicht mehr übersehen werden.

- Manche Kritiker meinen auch, es gebe bereits genug audiovisuelle Medien, die für Unterricht, Bildung und Gemeindearbeit zur Verfügung stünden. Videotechnik sei daher entbehrlicher Luxus, Videoarbeit nicht nötig. Die geringen finanziellen Mittel müßten zugunsten anderer Prioritäten verwendet werden.

Alle diese Bedenken, Einwände und Vorbehalte sind ernst zu nehmen, weil sie ihr Richtiges haben. Gegen diese Erfahrungen und Ansichten können aber auch Argumente angeführt werden. 


\section{Video als audiovisuelles Gruppenmedium}

$\mathrm{Da} ß$ sich Video als audiovisuelles Medium für kleinere Gruppen bis etwa 15 Teilnehmern sowohl als Abspiel- als auch als Ausdrucksmedium bewährt, haben die letzten Jahre immer deutlicher gezeigt.

\subsection{Video als audiovisuelles Medium}

Wie bei anderen AV-Medien, so hängt auch die Wirkung von Video vor allem von drei Faktoren ab: Von der Totalsprache des Mediums, von einer dramaturgischen Verarbeitung des Stoffes und von der Psychodynamik des Filmerlebens.

a. Totalsprache - Der Mensch kann, wie es die Pyramide des Bewußtseins zeigt, auf mehreren Ebenen angerührt betroffen werden:

- Auf der Ebene des reflexen Bewußtseins handelt es sich vorwiegend um kognitivrationale Vorgänge: Um Prozesse im Bereich der Wahrnehmung, des Denkens und der Gedächtnisleistung. Im Vordergrund steht die Aneignung von Faktenwissen; Überblick und Einblick in Sachverhalte wird ebenfalls gewährt; es wird beurteilt und entschieden, zum Handeln geschritten. Abläufe auf dieser Bewußtseinsebene sind weithin steuer- und kontrollierbar.

- Die emotional-affektive Dimension, der unterbewußte Bereich, umfaßt das weite Feld des Gefallens oder Mißfallens, der Anmutungen und Gefühle, der Interessen, Einstellungen, Haltungen und Werte. Wird ein Teilnehmer daraufhin angesprochen, so treten Emotionen und Affekte zutage. Bewußtseinsvorgänge auf dieser Ebene sind nicht mehr problemlos durchschaubar und lenkbar, weil sie sich zum großen Teil in den unterbewußten und unbewußten Schichten der Persönlichkeit abspielen.

- Zur unbewußten Ebene gehören alle Erfahrungen und Erlebnisse, die wir im Laufe unserer individuellen Lebensgeschichte durchgemacht haben. Sie sind mit allen positiven wie negativen Folgen zum Bestandteil unserer Persönlichkeit geworden. Unerreichbar tief im Unterbewußtsein und im Unbewußten schlummern sie. Obwohl sie unser Verhalten mitbestimmen und die Persönlichkeit prägen, lassen sie sich nur in geringem Umfang erhellen, etwa in einer psychotherapeutischen Behandlung. Aber sie lassen sich nicht mehr vollständig ins Bewußtsein heraufholen.

Skizze: Schichten der Ansprechbarkeit/Kegel des Bewußtseins

Kegel des Bewußtseins

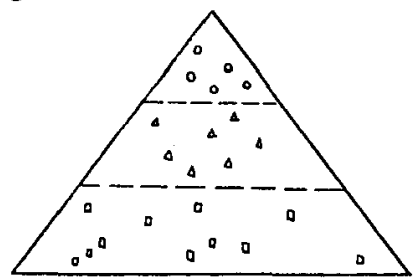

- Reflexes Bewußtsein

$\triangle$ Emotionale Schicht/Unterbewußtsein

- Unbewußte Ebene
Ansprechbarkeit durch AV-Medien

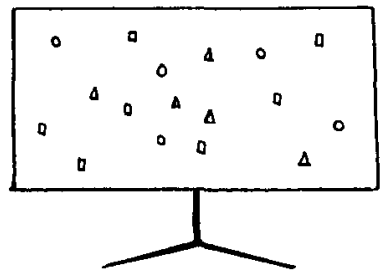

Audiovisuelle Medien aktivieren Erfahrungen, Gefühle und Erkenntnisse der Rezipienten. Die Teilnehmer werden durch die Totalsprache der AV-Medien und durch die Identifikation ganzheitlich angesprochen. 
Die „Totalsperre“ von Video berührt den Menschen in allen Persönlichkeitsschichten, weil sie sich aller Ausdrucksmittel bedient: Mimik und Gestik der Akteure, Geräusche, Sprache und Musik, Farbe, Bewegung, wechselnde Schauplätze und dramaturgische Effekte. Dadurch werden Aufmerksamkeit erregt und Spannungen erzeugt, sowie multisensorische Wahrnehmungen ermöglicht, was sich bei den Zuschauern in einer Steigerung der Wirkung niederschlägt. Die Totalsprache führt zu einer ganzheitlichen Ansprache, die nicht nur Verstand und Willen betrifft, sondern auch den emotionalen Bereich und die unbewußten Schichten erfaßt.

b. Schaffung von Erlebnissen: Voraussetzung hierfür ist eine gelungene dramaturgische Verarbeitung des Videostoffes: Eine Idee entwickelt sich; der Inhalt nimmt Gestalt an; die Story gerät in Bewegung und wird anschaulich aufgelöst. Dabei wechseln sich Phasen der Spannung und Entspannung immer wieder ab. So kommt es in den meisten Videoproduktionen zu einem Konflikt: Der Hauptdarsteller und sein Gegner stehen sich gegenüber; oft sind es aber auch Meinungen, Ansichten, politische Programme oder Weltanschauungen, die These oder Antithese bilden. Durch die Darstellung elementarer Empfindungen (zum Beispiel Freude, Liebe, Erfolg, Streben nach Sicherheit beziehungsweise Trauer, $\mathrm{Ha}$, Niederlage oder Angst) werden die erforderliche Aufmerksamkeit und Beteiligung des Publikums, ihre Zu- und Abneigung mit dem Videogeschehen, insbesondere mit den Handlungsträgern erreicht. Zuschauer fühlen sich angesprochen, in Entscheidungssituationen gestellt, zur Auseinandersetzung herausgefordert.

c. Das Filmerleben: Die Psychodynamik des Filmerlebens, von Kinovorführungen her hinlänglich bekannt, behält ihre Bedeutung auch für den Videobereich. Dabei tritt die Projektion beim Zuschauer dann auf, wenn Videobilder in ihm psychische Energien auslösen. Am besten gelingt dies mit Darstellern, die das tun, was der Betrachter sich wünscht, wovon er träumt. Je mehr ungestillte Sehnsüchte ein Mensch besitzt, um so größer ist seine Projektionsbereitschaft. Die Introjektion dagegen führt zu einer „Einseelung“ von Vorgängen, die sich auf der Leinwand abspielen: Das Aussehen eines Hauptdarstellers oder seine Handlungsweise werden vom Zuschauer aufgenommen und versickern in seiner Psyche. Das kann so weit gehen, daß der Fan seinen Star in Erscheinung und Verhalten nachahmt. Projektion und Inprojektion zusammen ergeben die Identifikation: Es kommt zu einer, zumindest partiellen Gleichschaltung der Gefühls- und Gedankenwelt des Zuschauers mit den Vorgängen auf dem Bildschirm. Der Kampf zwischen Gut und Böse, der Widerstreit der Meinungen, die unterschiedlichen Entscheidungen und Handlungen wie sie sich in bewegten Videobildern abspielen, werden zum Psychodrama: Es kommt zur innerseelischen Auseinandersetzung zwischen den im Menschen sich streitenden zustimmenden und ablehnenden Kräften.

\subsection{Video als kommunikativ-didaktisches Gruppenmedium}

Vier Elemente, die sich gegenseitig beeinflussen, treten bei der Verwendung von Video in Unterricht und Bildung, in kirchlicher Gemeindearbeit und sozial-kommunikativen Prozessen in Erscheinung:

a. Die Gruppe: Es handelt sich hierbei um eine Gemeinschaft, die aus einer überschaubaren Anzahl von Mitgliedern besteht, um eine Schulklasse, Jugendgruppe oder um eine Bürgerinitiative. Normalerweise kennen sich die Mitglieder einer Gruppe; 
zumindest werden sie von gemeinsamen Interessen oder Zielen zusammengeführt und geleitet. Bei Einsatz und Auswertung von Videoproduktionen spielen deshalb auch gruppendynamische Vorgänge eine wichtige Rolle: Das Verhalten des Einzelnen innerhalb der Gruppe, die Beziehungen der Gruppenmitglieder zueinander, interpersonale Konflikte, Autoritätsfragen et cetera. Zur Arbeit mit Video gehört deshalb nicht nur die Vorführung, sondern auch das, was das Videoband in der Gruppe an Interaktionen auslöst.

b. Der Gruppenleiter: Er kennt seine Gruppe, weiß sich ihr verbunden und sorgt, oft in Zusammenarbeit mit den Mitgliedern, für die Auswahl der Videokassetten. Bei der Arbeit mit Video kann die Rolle des Gruppenleiters ihren autoritären Akzent zugunsten eines eherpartnerschaftlich-orientiertenUmgangsstils verlieren: Lernende und Lehrende, Interessierte und Fachleute treten den Problemen und Themen gleichermaßen gegenüber; sie stehen auf derselben Ebene der kritischen Auseinandersetzung mit den dargestellten Inhalten des Videofilms. Keine Frage auch, daß die Verwendung von Video den Gruppenführer oft von seinen Informations- und Präsentationspflichten entlastet und ihn für erzieherisch-pädagogische Aufgaben freistellt: Für die Beobachtung der Gruppenatmosphäre, für eine verstärkte Betreuung einzelner Mitglieder und für die Vorbereitung der Auswertungsarbeit.

c. Das Medium: Die Art und Weise wie Alltagsereignisse oder Bildungsinhalte vom Publikum wahrgenommen, erfahren und erlebt werden vollzieht sich auf jeweils andere Weise über das Buch, einen Vortrag des Lehrers, über die Lichtbildreihe oder das Telefon, über den 16-mm-Farbtonfilm oder das Videoband. Der Anschaulichkeit und Aussagekraft gut gestalteter Videographien kann sich das Publikum nur schwer entziehen, zumal sie zunehmend eigene akustische, visuelle und dramaturgische Gestaltungsformen aufweisen: Mehr Großaufnahmen, die Nähe und Intimität suggerieren, und weniger Totalen; überraschende Verbindungen von Real- und Trickszenen; alle Arten von Ein- Aus- und Überblendungen; gelungene Farbkombinationen sowie die Verwendung verfremdeter Sprach- und Musikelemente. Die Möglichkeiten mit der Videotechnik zu experimentieren und neue Ausdrucks- und Darstellungsformen zu finden sind schier unbegrenzt und noch lange nicht ausgeschöpft.

d. Der Bildungs- und Aktionsprozeß: Erziehungs- und Bildungsbemühungen, Maßnahmen zur Sensibilisierung für Problembewußtsein der Öffentlichkeit oder Anregungen zu Initiativen in kirchlichen Gemeinden sind lebendige Vorgänge. Videoproduktionen können als Elemente mit unterschiedlichen Funktionen an verschiedenen Stellen schulischer Lernprozesse, kirchlicher Bildungsbemühungen oder demokratischer Initiativen eingesetzt werden. Um eine erfolgversprechende Auswahl und effektive Verwendung sicherzustellen, sollten folgende Gesichtspunkte beachtet werden:

- Die Qualität der Videoproduktionen ist von zwei Faktoren abhängig: Von der technischen Perfektion der Kopie und von der künstlerischen Gestaltung des Inhalts. Die Bildreproduktion sollte in Schnitt, Brillanz der Farben und Schärfe einwandfrei, die Tonwiedergabe unverzerrt und verständlich sein. Noch wichtiger ist allerdings die künstlerisch-formale Gestaltung der Inhalte: Die dargestellte Problematik muß sich den dramaturgischen Gesetzen entsp rechend überlegt und spannungsreich entwikkeln und eine wirkliche Aussagedichte erreichen; wenn auch nicht gleich auf Anhieb, so müssen Videoproduktionen die Zuschauer doch packen und über eine vordergründig-greifbare Gegenständlichkeit hinausführen. Dies geschieht am ehesten dann, wenn Videoprogramme Einblick in unbekannte Seiten der Wirklichkeit geben, 
wenn sie ungeahnte Zusammenhänge aufdecken, wenn sie zum Entdecken und Weiterfragen anregen, wenn sie Grundwerte auf anschauliche und spannende Weise zum Ausdruck bringen, wenn sie Orientierung für das Verhalten geben und geplante Aktionen begünstigen.

- Mediendidaktisch sinnvoll ist es, nur solche Videokassetten einzusetzen, deren Thematik und Gestaltungsform einen Bezug zum Leben der Adressaten haben. Dabei sollte der Videoeinsatz stets lehrplanbezogen sein, das heißt, er darf den Zielen (Absichten, Leitideen) etwa der religiösen Erziehung oder kirchlicher Verkündigungsbemühungen nicht zuwiderlaufen. Jedenfalls ist die Funktion, die eine Videoproduktion in der konkreten Verwendungssituation haben soll, mit den angestrebten Bildungs- und Aktionszielen in Übereinstimmung zu bringen.

- Videoprogramme in Lehr- und Lernprozessen, und dazu zählen auch kirchliche Gemeindearbeit und christliche Evangelisationsbemühungen, müssen stets auch in den Bildungsablauf eingebaut werden: So kommt es in der Eingangsphase von Bildungsveranstaltungen darauf an, das Vorwissen und die Voreinstellungen der Teilnehmer zu erheben. Ebenfalls ist es hilfreich, ihre Motivation zu fördern und sie mit dem Thema zu konfrontieren. Ziel sollte es sein, dem Zuschauer ein Problembewußtsein zu vermitteln, damit ein selbständiger Denk- und Aktionsprozeß in Gang kommen kann. Hierfür eignen sich vor allem solche Videofilme, die Fragen provozieren und Probleme stellen ohne sie zu lösen. Andere bieten die Vergleiche an und zeigen kontroverse Standpunkte auf oder geben Impulse zum Weiterdenken und Weiterarbeiten. In der Phase der Darbietung, Differenzierung und Erarbeitung werden Tatbestände dargestellt, Vorgänge erläutert, Grundbegriffe erklärt, wichtige Erkenntnisse vermittelt und Einblick in Zusammenhänge gewährt. Hierfür kommen Videofilme in Betracht, die Vorgänge dokumentieren und Sachverhalte strukturieren. Aber auch veranschaulichende Filme oder konkrete Fallbeispiele tragen dazu bei, bereits vorhandenes Wissen zu ergänzen und zu vertiefen.

- Die Botschaft, die Aussage der Videoprogramme muß themenorientiert, der Struktur des Bildungsinhaltes entsprechen und sachlich richtig sein. Videofilme, die biblische Inhalte zum Gegenstand haben, sollen mehr sein als nur Anschauungsmittel oder Illustrationen, die sich in historischen, kulturgeschichtlichen oder geographischen Details erschöpfen. Soll eine Videoproduktion der Eigenart biblischer Texte und Erfahrungen gerecht werden, so muß sie die kerygmatische Intention(Verkündigungsabsicht) zum Ausdruck bringen und in der Lage sein, das in der Überlieferung verborgene Glaubensgut darzustellen. Vom Videoprogramm zu biblischen Themen wird gewissermaßen eine audiovisuelle Exegese gefordert, die über Ohren und Augen zur Sinnmitte der Botschaft Jesu führt.

- Auch der Adressatenkreis muß beim Einsatz von Videokassetten hinsichtlich seines Erfahrungs- und Verstehenshorizontes berücksichtigt werden. Bei der Verwendung von Videokassetten ist deshalb immer auch die „Empfangssituation“ der Zielgruppe, die zugleich eine Lern- und Kommunikationssituation darstellt, zu beachten: Alter, Reifegrad, Geschlecht, Vorbildung und vorhandene Erfahrungen auf einschlägigem Gebiet sowie die Lebensumstände. Es gilt immer wieder zu fragen: Welche Bedeutung hat das Videoband für die Gruppe? Inwiefern beherrschen die Mitglieder bereits Methoden und Techniken, die eine sinnvolle Auswertung der Videokassetten ermöglichen? Können die Teilnehmer beispielsweise schon das wesentliche einer Bildfolge erfassen und sind sie in der Lage, eine videographische Darstellung zu protokollieren? 
- Bevor Videofilme eingesetzt werden, ist schließlich auch noch zu überlegen, wie ihre Auswertung erfolgen soll.

Durch die Entscheidung für eine unpassende Methode wird der Effekt des Mediums in der Bildungssituation oft verhindert. Informationsdichte und Länge vieler Videoangebote erfordern methodische Überlegungen, beispielsweise die Verteilung von Beobachtungsaufträgen bereits vor der Vorführung. Es kann sich auch anbieten, den Film in mehreren Abschnitten zu analysieren (Sequenzmethode). Bei kurzen Laufzeiten sollte das Programm nach mehrmaliger Vorführung besprochen werden (Frequenzmethode). Methodische Einsatz- und Auswertungsweisen sind keine bloß „technischen“Zugaben, sie schließen vielmehr Inhalte und Medien für die Bildungsund Kommunikationsziele auf. Methodische Verfahren stellen das Handwerkszeug dar, um Ziele, Inhalte und Medien so aufeinander zu beziehen, daß sie ihre jeweilige Funktion innerhalb eines Vorhabens erfüllen können.

- Videoproduktionen treten je nach Verwendungszusammenhang im wesentlichen in zwei didaktischen Funktionen auf: Entweder reichern die Filme herkömmliche Medien und Bildungsinhalte an (Enrichment-Funktion). In diesem Falle bleibt der Unterricht „lehrerzentriert“. Videoangebote unterstützen als Hilfsmittel die Steuerungsfunktion des Bildungsmultiplikators, beeinflussen den Bildungsvorgang insgesamt aber nicht primär. Oder Videoprogramme werden in der Funktion des Directteaching-ware eingesetzt. In diesem Fall übernehmen sie in einer „selbstlehrenden Funktion" die Rolle des Lehrers; dadurch wird der Bildungsprozeß „medienzentriert". Hierbei handelt es sich meist um „geschlossene" Lehrprogramme, die entweder monomedial angelegt sind, zum Beispiel als Englisch-Kurse mit Sprachlehranlagen. Oder sie sind multimedial im Sinne von Medienpaketen oder Medienverbundsystemen, wie zum Beispiel die 13-teilige Serie „Warum Christen glauben“. Dem Bildungsleiter verbleiben nach wie vor Lenkungs- und Deutungsfunktionen und er ist als Organisator und Supervisor des Bildungsgeschehens verantwortlich für die Erstellung und Umsetzung der Lehr- und Lernangebote; als Diagnostiker wird er auch immer wieder herauszufinden versuchen, welcher Lernende oder welche Gruppe besonderer Zuwendung bedarf und gefördert werden muß.

\section{Kommunikative und medienpädagogische Aspekte praktischer Videoarbeit}

Hier wird nur knapp auf einige Gesichtspunkte eingegangen, die mit der Verbreitung und Verwendung des Videosystems als einem kommunikativen und medienpädagogischen Mittel zusammenhängen:

a. Mit Video haben Individualisierung und Privatisierung des Filmkonsums ihren vorläufigen Höhepunkt und zugleich einen gewissen Abschluß erreicht. Nach der Erfindung bewegter Bilder und ihrer Verbreitung um die Jahrhundertwende mußte das Publikum zunächst auf den Jahrmarkt oder ins Kino gehen: Dort konnte es zu bestimmten Zeiten Filme sehen, die andere hergestellt und für eine Vorführung ausgewählt hatten. Mit dem Auftauchen des Fernsehens und seiner Entwicklung zum Massenmedium in atemberaubend kurzer Zeit zu Beginn der 50er Jahre, kamen von Redakteuren betreute und von Programmachern zusammengestellte Laufbilder zu den Menschen und in die Wohnzimmer der Familien. Als neues Trägersystem für Ton und Bild ermöglicht nunmehr Video nicht nur eine auf individuelle Wünsche abgestimmte Programmwahl und den von Kinovorstellungen und Fernsehaus- 
strahlung zeitlich und räumlich unabhängigen Konsum von Filmen auch für Kinder und Jugendliche, sondern darüber hinaus die relativ kostengünstigste Aufzeichnung, Überspielung, Speicherung oder Selbstherstellung von Filmen.

b. Gegenüber dieser Entwicklung nehmen die einen eine kritische bis ablehnende Haltung ein, die sich etwa in folgenden Bedenken äußert: Übermäßiger Medienkonsum führe zu Überreizung und Nervosität, zu psychischer Desintegration und verringertem Selbstbewußtsein, zum Abbau zwischenmenschlicher Kontakte und zu wachsender Vereinsamung, zur Vernichtung der Kreativität. Es wird von Drogenwirkung und Videosucht gesprochen und davon, daß vor allem (kleinere) Kinder die Scheinwelt der Medien mit der Realität des Lebens vermengten und sich deshalb im konkreten Alltag nicht mehr zurechtfänden. Hingewiesen wird insbesondere immer wieder auf unabsehbare Folgen für die psychische, moralische und soziale Entwicklung der Heranwachsenden, die das regelmäßige Ansehen von Action- Horror- und Sexfilmen haben könne. Andere wieder sehen in der Videotechnik, bei aller kritischen Reserve gegenüber Mißbrauch und Auswüchsen, einen starken Wachstumsfaktor für die Wirtschaft. Sie halten eine konsequente audiovisuelle Abstinenz für undurchführbar, da wir alle - einschließlich der (kleineren) Kinder - von Kino, Fernsehen und Neuen Medien umgeben sind. Ein striktes Verbot von Fernsehsendungen und Videofilmen würde für Heranwachsende einer sozialen Askese gleichkommen mit unzweifelbaren Schäden für ihre gesamtmenschliche Entwicklung. In bewegten Bildern, wie immer sie auch technisch vermittelt werden, sehen sie für Kinder und Jugendliche eine unverzichtbare Quelle der Information, Bildung und Unterhaltung. Die visuellen Medien bilden in ihren Augen einen hochbewerteten Gesprächsstoff in der Gruppe der Gleichaltrigen und seien zugleich Anregungen für phantasiereiche Tätigkeiten. Um den jedoch unbestrittenen Gefahren zu begegnen, halten sie daher eine „Medienalphabetisierung“ für notwendig, die Kinder möglichst früh zu einem maßvollen und kritischen Umgang mit den modernen Kommunikations- und Darstellungsmedien erzieht.

c. Weder ein Rückfall in eine reine Bewahrpädagogik, noch die Resignation vor der realen Entwicklung, auch bloße Polemik gegen die Neuen-Medien oder sogar völlige Medienabstinenz scheinen mir in der Tat nicht geeignet, auf einen kritischen und verantwortungsbewußten Umgang mit audiovisuellen Medien, insbesondere auch mit Video, vorzubereiten. Wenn die Arbeit in und mit den Medien nicht von innen her geschieht, bleibt alles andere nur äußerliches Bemühen, können auch gesetzgeberische Maßnahmen gegebenenfalls nur das Schlimmste verhüten und nur den Rahmen für Mißbräuche abstecken. Bischof Georg Moser, Vorsitzender der Publizistischen Kommission der Deutschen Bischofskonferenz, verlangt angesichts der konkreten Situation im Videobereich deshalb konsequenterweise „Zugangsregelungen für Kinder und Jugendliche, die dem Gesetz zum Schutz der Jugend in der Öffentlichkeit für öffentliche Filmveranstaltungen entsprechen“. Darüber hinaus fordert er „die zuständigen Behörden, insbesondere die Kultusministerien der Länder sowie die Bildungseinrichtungen auf, durch systematische Förderung der Medienpädagogik wirksame Hilfen zur Bewältigung und zur sinnvollen Nutzung der Medien zu leisten“. An Eltern und Erzieher appelliert er ferner, ,ihre medienerzieherische Verantwortung für die ihnen anvertrauten Kinder zu erkennen und verstärkt wahrzunehmen “' . Letztlich führt auch hier nur positives Tun weiter, Schimpfen und Angstmachen allein nützen niemandem. Gerade die Integration von Video in soziale Aktionen, in didaktisch-strukturierte Bildungsbemühungen oder kommunikative Prozesse ermöglicht den sachgerechten Umgang mit dem Medium und arbeitet 
damit einer bloß passiven Konsumhaltung entgegen. Medienpsychologie und Medienwirkungsforschung haben darüber hinaus nachgewiesen, daß Filme eine stark aktivierende Kraft haben: $\mathrm{Daß}$ sie den psychomotorischen Apparat beleben und zur Auseinandersetzung, Wertung, Stellungnahme und Entscheidung herausfordern. Voraussetzung hierfür ist allerdings, $\mathrm{da} ß$ mit dem Videomaterial gearbeitet wird, daß Videofilme nicht als bloße Konsumware oder als „Selbstläufer" eingesetzt werden, ohne einführende Hinweise und ohne nachfolgende Aufarbeitung. In der Praxis vor Ort zeigt sich immer wieder, daß Medien dann am effektivsten wirken, wenn über das Dargebotene intensive Gespräche geführt werden oder wenn ein Bezug zu Lebenssituationen und Existenzfragen der Beteiligten hergestellt wird.

Damit komme ich zu einer letzten Anmerkung: Bertold Brecht hatte schon 1932 in den „Schriften zur Literatur und Kunst“ den Vorschlag der Umfunktionierung des Rundfunks gemacht, womit allerdings das Radiogemeint war: „Der Rundfunk ist aus einem Distributionsapparat in einen Kommunikationsapparat zu verwandeln. Der Rundfunk wäre der denkbar großartigste Kommunikationsapparat des öffentlichen Lebens, ein ungeheures Kanalsystem. Er wäre es, wenn er nicht nur auszusenden verstünde, sondern auch zu empfangen, und den Zuhörer nicht zu isolieren, sondern mit ihm eine Beziehung aufzunehmen. Der Rundfunk müßte demnach ... den Hörer als Lieferanten organisieren" (Band I, Frankfurt: 1967, 134, Suhrkamp-Verlag). Das Videosystem scheint hierfür besonders geeignet zu sein: Es ermöglicht den Schritt über einen eher „passiven“ Konsum vorgefertigter Medien hinaus hin zur „aktiven“ Medienarbeit. Mit der Videotechnik entwickeln und realisieren Teilnehmer (Schulklassen, Jugendgruppen, Interessengemeinschaften) eigene audiovisuelle Produkte. Aktive Videoarbeit hat das Ziel, die Trennung zwischen Medientheorie und Medienpraxis, von Wissen und Handeln aufzuheben. Indem die Einrichtungen Schule, Erwachsenenbildung und Kirchengemeinde denen das audiovisuelle Medium Video als Ausdrucksmittel anbieten, die nicht mit den traditionellen Mitteln der Kommunikation wie dem Buch oder der freien Rede umzugehen gelernt haben, ist Videoarbeit zugleich Medienkunde. Videogruppen, die selbst Medien herstellen, leisten einen Beitrag zur Entmystifizierung von Kino und (Kabel-) Fernsehen: Aus der eigenen Erfahrung im Umgang mit Video erhalten sie Einsicht in die Möglichkeiten und Gesetzmäßigkeiten der Filmherstellung und Fernsehproduktion. Themenstellung für eine „Videoarbeit vor Ort" sollte vor allem aus dem konkreten Lebensumfeld der Teilnehmer entwickelt sein und adressatenorientiert aufbereitet werden. Insofern diese Arbeit an lokale oder regionale Ereignisse und Zustände anknüpft und Stadtteil - oder Minderheiten sowie Gruppenprobleme aufgreift, wird der Umgang mit dem Medium Video auch zur sozialen und politischen Bildungsarbeit. Videogruppen leisten einen Beitrag zu einer neuen Form der Öffentlichkeit, sofern sie sich nicht nach außen hin abschotten. Das ist aber nahezu unmöglich, da das Produkt Videofilm für ein wie immer geartetes Publikum hergestellt wird. Wenn die Videogruppen und Bürgerinitiativen an Ort und Stelle eine verstärkte Kooperation betreiben, dann bauen sie eine basisnahe Kommunikation in kirchlichem Gemeindebereich und in der politisch-sozialen Öffentlichkeit auf. Damit verbessern sie das Problembewußtsein und die Qualität des Dialogs in der Gesellschaft. Die Aktivierung der Bürger im lokalen, also einigermaßen überschaubaren Bereich, ist einer der bedeutendsten aktuellen gesellschaftspolitischen Prozesse. Aktivierung meint in dem Zusammenhang eine zunehmende Selbstbestimmung und Selbstverantwortung in persönlichen und lokalen Angelegenheiten. In diesem Zusammenhang kann Video als audiovisuelles Ausdrucks- und Kommunikationsmittel wertvolle Dienste leisten! 


\section{Anmerkung}

1 vgl. Bischof Georg Moser: Herausforderung durch Videokassetten, Erklärung des Vorsitzenden der Publizistischen Kommission der Deutschen Bischofskonferenz vom 30. August 1983, Zentralstelle für Medien, Bonn. 


\section{SUMMARY}

Anton Täubl, in his article, looks at the present situation of the video medium for educational institutions in the Federal Republic of Germany. Compiling first the pros and cons of the current debate, he finally joins the party supporting the use of video. From that angle, the attributes of video as an audio-visual group medium are described in detail, stressing mainly the educational aspects.

\section{RÉSUMÉ}

Dans son article, Anton Täubl examine la situation actuelle de la vidéo dans les instituts de formation de la République Fédérale d'Allemagne. Il commence par réunir les arguments pour et contre pour finalement se ranger du côté des tenants de la vidéo. Avec cette attitute positive, il examine alors en détail les qualités de la vidéo utilisée comme moyen de groupe, en insistant sur la valeur de la vidéo comme outil d'éducation.

\section{RESUMEN}

En este artículo, Anton Täubl examina la situación actual del video en los institutos de formación en la República Federal Alemana, reuniendo primeramente los argumentos en favor y en contra de esta utilización, pero finalmente el mismo autor se pone del lado de quienes favorecen el uso del video. Con esta actitud positiva luego él realiza un examen detallado de las cualidades del video utilizado como media de grupo, insistiendo en el valor de este instrumento en el campo de la educación. 